\title{
Characteristics of Pediatric Glaucoma in a Latin American Reference Center
}

\author{
Catalina Saavedra ${ }^{1}$, Hernan A Rios ${ }^{2}$, Sandra Belalcazar ${ }^{3}$, Shirley M Rosenstiehl ${ }^{4}$
}

\begin{abstract}
Aim:To describe the clinical and epidemiological characteristics of children with pediatric glaucoma (PG) treated in an ophthalmologic national reference center.

Materials and methods: A retrospective study of patients diagnosed with PG in a national ophthalmologic reference center was made, between 2005 and 2015. Clinical findings, type of treatment, and the follow-up were evaluated.

Results: A total of 89 patients (145 eyes) were included. The median age of diagnosis was 2.0 years. The most frequent type of glaucoma was primary PG with $67.4 \%$ of affected patients, primary congenital glaucoma (PCG) being more frequent (69 eyes) than juvenile open-angle glaucoma (JOAG 36 eyes). Secondary PG accounted for $32.6 \%$ of the cases (40 eyes). At least one surgical procedure was needed in $56.6 \%$ of all studied eyes, and $10.7 \%$ of eyes had more than two surgical procedures. Even more, eyes with PCG had surgery in $88.4 \%$ of cases. On the contrary, eyes with JOAG did not require surgery. In the last assessment, the distribution of cases according visual acuity did not show differences. However, it is important to note that patients with secondary PG maintained a good vision only in $17.9 \%$ of cases.

Conclusion: Pediatric glaucoma is a heterogeneous group of diseases, and due to its low incidence, descriptive reports of large cohorts are not available. This study has a well-detailed report of PG characteristics in a national reference center. The frequency of JOAG in the present study was significantly higher than that reported in other studies. Also, clinical characteristics of all glaucoma described have some differences from data published.

Clinical significance: There are few studies that describe characteristics of PG. This study is an important tool to analyze the characteristics of $P G$ in an effort to better know the disease.

Keywords: Pediatric glaucoma, Retrospective study, Surgical management, Treatment and follow-up of glaucoma.

Journal of Current Glaucoma Practice (2020): 10.5005/jp-journals-10078-1267
\end{abstract}

\section{INTRODUCTION}

Pediatric glaucoma (PG) is a heterogeneous group of diseases which all share ocular hypertension pathways and pressure-related damage to ocular structures. ${ }^{1}$ Primary congenital glaucoma (PCG) is the most frequent type, with an incidence of 1:10,000 to 68,000 live births. ${ }^{2,3}$ Due to the low incidence of this disease, descriptive reports of large cohorts are not available. ${ }^{2}$ In our country, one descriptive study of PG was published in 1999; clinical and epidemiological characteristics of only 34 children diagnosed with primary or secondary PG were reported. ${ }^{4}$

Different clinical studies suggest intraocular pressure (IOP) as the main prognostic factor to control the progression of the disease and irreversible damage on the cornea and optic nerve, preventing definitive blindness. ${ }^{5}$ Approximately, $8 \%$ of blindness cases in pediatric patients have been attributed to PG. ${ }^{6}$ Timely and correct diagnosis of glaucoma is essential to optimize visual outcomes and to guide appropriate treatment in children.

This study describes the clinical and epidemiological characteristics of children with PG treated in an ophthalmologic national reference center. The frequency of different types of PG, associated conditions, systemic pathologies, and the response to medical and surgical intervention are also reviewed. This study provides relevant information on the clinical presentation and behavior of the disease in a developing nation.

\section{Materials and Methods}

This was a retrospective study of patients diagnosed with PG in a national ophthalmologic reference center, between 2005 and 2015.
1,2,4Fundación Oftalmológica Nacional, Bogota, Colombia

${ }^{3}$ Glaucoma Department, Fundación Oftalmológica Nacional, Bogota, Colombia

Corresponding Author: Sandra Belalcazar, Glaucoma Department, Fundación Oftalmológica Nacional, Bogota, Colombia, Phone: +57 3102338983, e-mail: sbelalcazarrey@gmail.com

How to cite this article: Saavedra C, Rios HA, Belalcazar S, et al. Characteristics of Pediatric Glaucoma in a Latin American Reference Center. J Curr Glaucoma Pract 2020;14(1):10-15.

Source of support: Resources from the Research Department of Fundación Oftalmológica Nacional

Conflict of interest: None

Medical records from patients younger than 16 years old with at least 3 months of follow-up were reviewed. This study adhered to the tenets of the Declaration of Helsinki and was approved by the institutional review board of the center. Medical records with incomplete data were excluded. Clinical findings, type of treatment, response to treatment, and the follow-up were evaluated. Latest consensus by World Glaucoma Association was used to classify the type of PG. ${ }^{7}$ According to the consensus, PG is classified as primary or secondary. Primary PG is divided into PCG and Juvenile Open-angle Glaucoma (JOAG). Secondary PG can be subclassified into glaucoma associated with nonacquired ocular abnormalities, glaucoma associated with systemic diseases (or nonacquired syndromes), glaucoma associated with acquired conditions, and glaucoma following cataract surgery. ${ }^{7}$ Visual acuities unable to be 
assessed with Snellen charts were converted using the following convention: count fingers, 20/2,000; hand motions, 20/20,000 and light perception, 20/200,000. ${ }^{8}$ Visual acuity from non-verbal children medical records (Binocular Fixation Preference) was rounded to Snellen using the following convention: central, steady, and maintained was converted to visual acuity better than 20/50; no central, no steady, and no maintained was converted to worse than 20/200; and the patients who had only one of the criteria (central, steady, or maintained) were classified as visual acuity between $20 / 50$ and $20 / 200$ by Snellen. ${ }^{8}$ Data were analyzed with SPSS statistics program 21 (ser. 572110 343, SPSS, Inc., Chicago, IL).

\section{Results}

Medical records from patients diagnosed with PG in an ophthalmologic reference center between 2005 and 2015 were analyzed. A total of 89 patients (145 eyes) were included. Female cases corresponded to $49.4 \%$. The median age of diagnosis for the whole group of patients was 2.0 years [interquartile range (IQR): 9.0 years]. Patients with PCG were diagnosed at a median age of 0.5 years (IQR: 0.9 years), patients with secondary PG and JOAG were diagnosed at a later age of 4.5 years (IQR: 9.7 years) and 10.0 years (IQR: 5.5 years), respectively. The median follow-up time in all patients was 1.6 years (IQR: 2.3 years). The mean follow-up in PCG patients was 1.6 years (IQR: 1.4 years) and for JOAG patients was 1.3 years (IQR: 0.4 years). Secondary glaucoma patients had the longest follow-up time, 1.7 years (IQR: 3.6 years; Table 1).

The most frequent type of glaucoma was primary $P G$, accounted for $67.4 \%$ of affected patients, PCG being more frequent (42 patients, 69 eyes) than JOAG (18 patients, 36 eyes). Secondary PG accounted for $32.6 \%$ of the cases (29 patients, 40 eyes; Table 2). All JOAG patients had both eyes affected; conversely, almost a third of the PCG patients had unilateral presentation. However, secondary PG patients had a unilateral predilection (Table 2).

Most common secondary PG was glaucoma associated with nonacquired eye abnormalities and glaucoma associated with congenital cataract surgery. Glaucoma associated with dysgenesis of the anterior segment and glaucoma associated with congenital cataract surgery were reported as the most frequent etiologies, both accounted for $9.6 \%$ of all eyes. Patients with glaucoma associated with nonacquired systemic disease were found less frequently (Table 3).
At least one surgical procedure was needed in $56.6 \%$ of all studied eyes, and $10.7 \%$ of eyes had more than two surgical procedures. Even more, eyes with PCG had surgery in $88.4 \%$ of cases. On the contrary, eyes with JOAG did not require surgery (Table 4). In all reviewed cases, the procedure of choice for initial management was trabeculectomy, which was performed in $42.9 \%$ of the cases, followed by trabeculectomy plus trabeculotomy, Ahmed valve implant and trabeculotomy as a stand-alone procedure, with 29.9, 15.6, and 10.4\%, respectively (Fig. 1). Trabeculectomy was performed without the use of mitomycin in half of cases; however, mitomycin was not used in three cases of trabeculectomy plus trabeculotomy. Cyclodestruction was done only in one eye as initial procedure, because glaucoma was due to trauma.

In all studied cases, median of IOP at initial assessment was $16.0 \mathrm{~mm} \mathrm{Hg}$ (IQR: $10.5 \mathrm{~mm} \mathrm{Hg}$ ); however, the mean IOP at initial assessment reported in eyes with secondary PG was $20.5 \mathrm{~mm}$ Hg (IQR: $18.5 \mathrm{~mm} \mathrm{Hg}$ ). In the last assessment, the IOP average decreased in all types of glaucoma, with a greater decrease in eyes with secondary PG. Regarding cup-to-disk ratio, it remained quite similar during visits (Table 5). In the first visit, the mean number of topical medications for all eyes was 1.54. As expected, most eyes with JOAG and secondary PG were treated with at least one antiglaucomatous topical medication after the first visit. Most of the patients with JOAG (66.7\%) were treated with only one antiglaucomatous medication, prostaglandin analogs being most frequently used. Eyes with PCG reported no topical medication after the first visit in $27.5 \%$ of cases (Fig. 2).

At initial assessment, best-corrected visual acuity (BCVA) was better than $20 / 50$ in $43.1 \%$ of eyes, between $20 / 50$ to $20 / 200$ in $23.4 \%$ of eyes, and worse than $20 / 200$ in $33.6 \%$ of eyes. At the final assessment, the distribution of cases according BCVA did not show clear differences. However, it is important to note that patients with secondary PG maintained a good vision (better than 20/50) only in $17.9 \%$ of cases (Fig. 3).

\section{PCG Follow-up}

Sixty-nine eyes with PCG were diagnosed and managed. Primary management was surgical in most cases, trabeculectomy plus mitomycin $C$ being the most frequent procedure (24 eyes, 34.8\%). Trabeculotomy plus trabeculectomy was performed in 18 eyes (26.1\%). In total, $11.6 \%$ of eyes (eight eyes) had only trabeculotomy

Table 1: Gender distribution and age of diagnosis according to the type of glaucoma

\begin{tabular}{|c|c|c|c|c|c|c|}
\hline & \multicolumn{2}{|c|}{ Female } & \multicolumn{2}{|c|}{ Male } & \multirow{2}{*}{$\begin{array}{c}\begin{array}{c}\text { Median age of } \\
\text { diagnosis }\end{array} \\
\text { Years (IQR) }\end{array}$} & \multirow{2}{*}{$\begin{array}{l}\text { Median follow-up } \\
\text { time }\end{array}$} \\
\hline & Patients (eyes) & (\%) & Patients (eyes) & (\%) & & \\
\hline Primary congenital glaucoma & $23(34)$ & 25.8 & $19(35)$ & 21.4 & $0.5(0.9)$ & $1.6(1.4)$ \\
\hline Juvenile open-angle glaucoma & $6(12)$ & 6.7 & $12(24)$ & 13.5 & $10.0(5.5)$ & $1.3(0.4)$ \\
\hline Secondary pediatric glaucoma & $15(22)$ & 16.9 & $14(18)$ & 15.7 & $4.5(9.7)$ & $1.7(3.6)$ \\
\hline All pediatric glaucomas & $44(68)$ & 49.4 & $45(77)$ & 50.6 & $2.0(9.0)$ & $1.6(2.3)$ \\
\hline
\end{tabular}

$\mathrm{IQR}$, interquartile range

Table 2: Distribution of patients according to laterality

\begin{tabular}{lcll}
\hline & Unilateral (\%) & Bilateral (\%) & Total (\%) \\
\hline Primary congenital glaucoma & $15(16.9)$ & $27(30.3)$ & $42(47.2)$ \\
Juvenile open-angle glaucoma & $0(0.0)$ & $18(20.2)$ & $18(20.2)$ \\
Secondary pediatric glaucoma & $18(20.2)$ & $11(12.4)$ & $29(32.6)$ \\
Total (\%) & $33(37.1)$ & $56(62.9)$ & $89(100.0)$ \\
\hline
\end{tabular}


Pediatric Glaucoma in a Reference Center

Table 3: Diagnosis distribution

\begin{tabular}{llcc}
\hline & Patients & Eyes & Eyes (\%) \\
\hline Primary pediatric glaucoma & 60 & 105 & 72.4 \\
Primary congenital glaucoma & 42 & 69 & 47.6 \\
Juvenile open-angle glaucoma & 18 & 36 & 24.8 \\
Secondary pediatric glaucoma & 29 & 40 & 27.6 \\
Associated with nonacquired eye abnormalities & & & 4.8 \\
Dysgenesis of the anterior segment & 4 & 7 & 2.8 \\
Axenfeld-Rieger (anomaly and syndrome) & 2 & 4 & 2.1 \\
Aniridia & 2 & 3 & 2.8 \\
Peters (anomaly and syndrome) & 3 & 4 & 1.4 \\
Nevus of Ota & 1 & 2 & 0.7 \\
Associated with nonacquired systemic diseases & & & \\
Sturge Weber syndrome & 1 & 1 & 3.4 \\
Associated with acquired conditions & & 5 & 0.7 \\
Ocular trauma & 5 & 1 & 0.7 \\
Corticoid & 1 & 1 & 2.8 \\
Retinopathy of prematurity & 1 & 4 & 0.7 \\
Uveitis & 3 & 1 & 4.8 \\
Keratoplasty & 1 & 7 & 100.0 \\
Associated with congenital cataract & 5 & 145 &
\end{tabular}

Table 4: Number of surgeries per eye according to the type of glaucoma

\begin{tabular}{lccccc}
\hline & \multicolumn{5}{c}{ Number of surgeries } \\
\cline { 2 - 6 } & $0(\%)$ & $1(\%)$ & $2(\%)$ & $3(\%)$ & Total \\
\hline Primary congenital glaucoma & $11.6(8)$ & $63.8(44)$ & $21.7(15)$ & $2.9(2)$ & $100.0(69)$ \\
Juvenile open-angle glaucoma & $100.0(36)$ & $0.0(0)$ & $0.0(0)$ & $0.0(0)$ & $100.0(36)$ \\
Secondary pediatric glaucoma & $47.5(19)$ & $35.0(14)$ & $12.5(5)$ & $5.0(2)$ & $100.0(40)$ \\
All pediatric glaucomas & $43.4(63)$ & $40.0(48)$ & $13.8(20)$ & $2.8(4)$ & $100.0(145)$ \\
\hline
\end{tabular}

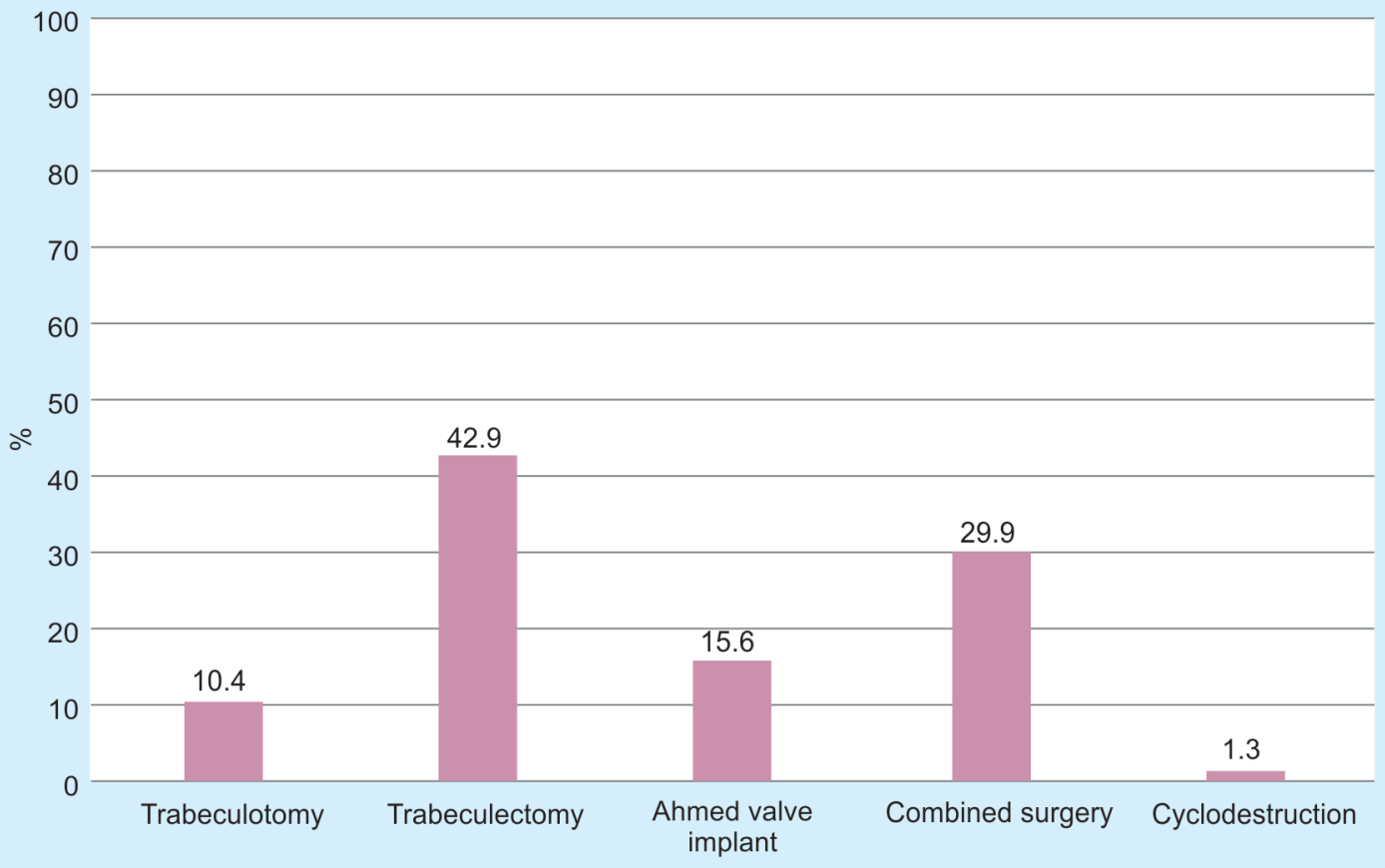

Fig. 1: Distribution of surgical initial management 
and 7 eyes (10.1\%) had an Ahmed valve implant. In total, $11.6 \%$ of cases (eight eyes) did not receive any type of procedure. One bilateral case had trabeculotomy plus trabeculectomy in one of his eyes, and the other eye had IOP controlled with topical medications and did not need procedure until the last assessment in the institution.

\section{JOAG Follow-up}

Thirty-six eyes were evaluated and all were managed and controlled with topical treatment. No surgical procedure was needed.

\section{Secondary PG Follow-up}

Forty eyes with secondary PG were diagnosed and managed. In total, $50.0 \%$ (20 eyes) of cases required surgery. Trabeculectomy plus mitomycin and Ahmed valve implant were the most frequently performed procedures. A case with Ahmed valve implant required

Table 5: Clinical characteristics and type of glaucoma in the study eyes

\begin{tabular}{|c|c|c|c|c|}
\hline & \multicolumn{2}{|c|}{ Median IOP (IQR) } & \multicolumn{2}{|c|}{$\begin{array}{c}\text { Median cup-to-disk } \\
\text { ratio (IQR) }\end{array}$} \\
\hline & Initial & Final & Initial & Final \\
\hline $\begin{array}{l}\text { Primary congenital } \\
\text { glaucoma }\end{array}$ & $16.0(11.0)$ & $13.0(4.0)$ & $0.6(0.4)$ & $0.5(0.4)$ \\
\hline $\begin{array}{l}\text { Juvenile open-angle } \\
\text { glaucoma }\end{array}$ & $13.0(6.8)$ & $12.0(2.3)$ & $0.7(0.2)$ & $0.8(0.2)$ \\
\hline $\begin{array}{l}\text { Secondary pediatric } \\
\text { glaucoma }\end{array}$ & $20.5(18.8)$ & $15.5(9.3)$ & $0.5(0.5)$ & $0.6(0.4)$ \\
\hline $\begin{array}{l}\text { All pediatric } \\
\text { glaucomas }\end{array}$ & $16.0(10.5)$ & $13.0(5.0)$ & $0.6(0.3)$ & $0.6(0.4)$ \\
\hline
\end{tabular}

later cyclodestruction procedure due to failed IOP control. A tube extension was also required in another case with Ahmed valve implant. Trabeculotomy plus trabeculectomy was conducted in five eyes; however, one case required a valve implant due to failed IOP control.

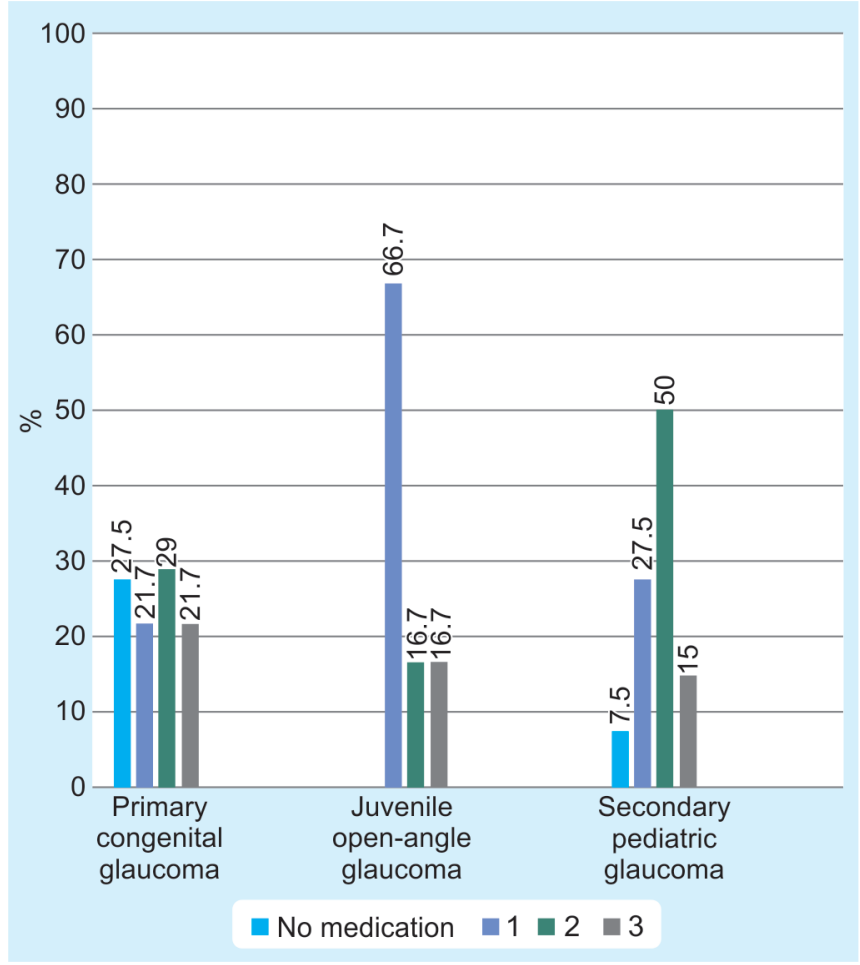

Fig. 2: Number of medications

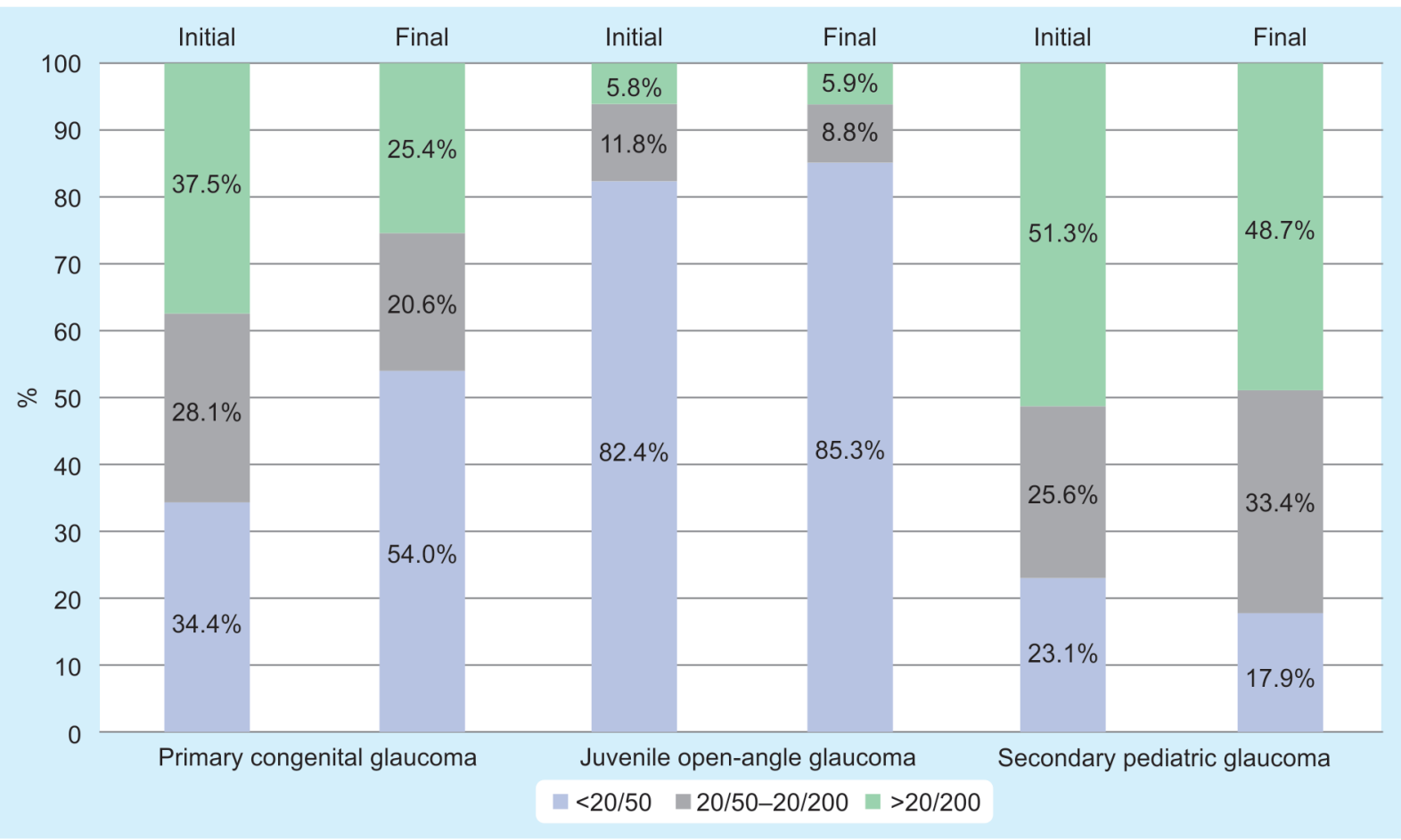

Fig. 3: Visual acuity 


\section{Complications}

Most of the complications occurred in the PCG cases. Complications reported were endophthalmitis, hyphema, tube displacement, vitreous prolapse, and leaking filtering bleb. Endophthalmitis was reported in a patient who received a scleral patch for scleral melting after trabeculotomy plus trabeculectomy. This patient developed phthisis bulbi on this eye.

\section{Discussion}

Pediatric glaucoma threatens the visual acuity of children. It may appear at any pediatric age. Late diagnosis may result in irreversible damage to optic nerve and visual function, affecting the development of these children. Therefore, the knowledge of the disease, early diagnosis and treatment are important to decrease long-term visual impairment. ${ }^{9-11}$

In this study, the majority of cases belonged to the PCG group, which is in accordance with that reported in other studies. The gender distribution was similar for PCG and secondary PG but not for JOAG.

There are few studies that describe the epidemiological characteristics of PG. The frequency of JOAG in the present study was significantly higher than that reported by Papadopoulos in the United Kingdom. A local study had a similar distribution according to the type of PG. ${ }^{4}$ However, Fung et al. found that secondary glaucoma was the most frequent type of PG, in $47.8 \%$ of cases regardless of race. The study by Fung et al. included 90 Hispanic subjects, and the most frequent type of glaucoma among this group was also secondary glaucoma. ${ }^{12}$ This finding contrasts with our results in which the most frequent type was PCG, corresponding to $47.2 \%$ of the cases. This could be explained by the multifactorial PCG etiology, not just the genetic factors. On the other side, there are many factors that are implied in PG diagnosis, making prevalence different worldwide. Reporting epidemiological and clinical factors of the disease in different populations may be a key to improve PG knowledge.

In our country, due to insurance affairs for health care issues, it may take long for these patients to get to the surgical treatment. This could be a condition affecting the visual prognosis of these patients, especially in patients with secondary glaucoma. Goniotomy is recommended as the first-line treatment when visualization through the cornea is adequate. In cases with corneal opacity, trabeculotomy is recommended. ${ }^{13}$ However, in some centers, trabeculotomy plus trabeculectomy is the first-line procedure in patients with PCG. Surgeons in this study had a predilection for trabeculectomy with mitomycin. It has been reported that trabeculotomy compared with the trabeculectomy plus trabeculotomy with mitomycin $C$ in cases of congenital glaucoma had similar outcomes. ${ }^{14}$ Compared with the study by Fung et al., JOAG eyes did not need any surgical procedure, because IOP was controlled with medications. ${ }^{12}$ The decision to treat pharmacologically and not surgically, patients with JOAG, was made given that these patients were older, had better cooperation with the ophthalmological examination, better adherence to topical treatment and mainly because the pathophysiology of the disease is different to PCG allowing better response to medical treatment.

The majority of the cases had surgical successful results with the stabilization of cup-to-disk ratio. Even more, after the surgical treatment, some cases had a change in the cup-to-disk ratio. This is considered possible due to normalization of IOP, high resilience of the connective tissue of the optic nerve, and the elasticity of the lamina cribrosa during development. ${ }^{15}$ Regarding surgical complications, it was found similar to those by Jalil et al. ${ }^{16}$
Due to certain limitations in the health-care system of our country, it is difficult to strictly follow-up these patients. It is considered that congenital glaucoma has a significant economic impact, mainly during the first year after diagnosis, due to many surgical procedures and examinations that must be done. ${ }^{17}$ Our country does not have demographic reports about incidence of PG. In our institution, it represented less than $2 \%$ of the pediatric patients seen during that period.

Finally, this study had several limitations. First of all, it is a retrospective study. Prospective studies for PG are difficult to perform because of its low incidence. Moreover, the follow-up in our practice may be difficult due to the changing access of the patients to health-care insurance.

In our country, follow-up of PG patients is difficult; however, some of them have been followed up for 8 years. This process is an important challenge not only for the ophthalmologist but also for parents and other caregivers. The treatment is mainly surgical and can be associated with complications during and after the procedures. The decisions taken to treat these patients have a critical impact on children, who will probably visit four or more generations of ophthalmologists. The visual prognosis of these patients depends on the time of presentation and immediate treatment, hence the importance of knowing the clinical and epidemiologic characteristics of this disease.

\section{Clinical Significance}

There are few studies that describe the characteristics of PG. The results from this study contribute to analyze the characteristics of PG in an effort to know the disease presentation and clinical and surgical management in a national reference center.

\section{References}

1. Hoguet A, Grajewski A, Hodapp E, et al. A retrospective survey of childhood glaucoma prevalence according to childhood glaucoma research network classification. Indian J Ophthalmol 2016;64(2):118. DOI: 10.4103/0301-4738.179716.

2. Moore DB, Tomkins $\mathrm{O}$, Ben-Zion I. A review of primary congenital glaucoma in the developing world. Surv Ophthalmol 2013;58(3):278285. DOI: 10.1016/j.survophthal.2012.11.003.

3. Taylor RH, Ainsworth JR, Evans AR, et al. The epidemiology of pediatric glaucoma: the Toronto experience. J AAPOS 1999;3(5):308-315. DOI: 10.1016/s1091-8531(99)70028-5.

4. Montezuma SR, Serrano JC. Glaucoma Pediátrico en la Fundación Oftalmológica de Santander. Bucaramanga (Santander): MEDUNAB; 1999. pp. 5-13.

5. Papadopoulos M, Cable N, Rahi J, et al. Study investigators. The British infantile and childhood glaucoma (BIG) eye study. Invest Ophthalmol Vis Sci 2007;48(9):4100-4106. DOI: 10.1167/iovs.06-1350.

6. Abdolrahimzadeh S, Fameli V, Mollo R, et al. Rare diseases leading to childhood glaucoma: Epidemiology, pathophysiogenesis, and management. Biomed Res Int 2015;2015:781294. DOI: $10.1155 / 2015 / 781294$.

7. Weinreb R, Papadopoulos M, Grigg J. Childhood glaucoma - World glaucoma association consensus series 9. Kugler Publ; 2013.

8. Lonngi M, Aguilar MC, Ríos HA, et al. Pediatric uveitis: experience in Colombia. Ocul Immunol Inflamm 2016(4):1-5. DOI: 10.3109/09273948.2016.1160129.

9. Sinha G, Patil B, Sihota R, et al. Visual field loss in primary congenital glaucoma. J Am Assoc Pediatr Ophthalmol Strabismus 2015;19(2):124129. DOI: 10.1016/j.jaapos.2014.12.008.

10. Zagora SL, Funnell CL, Martin FJ, et al. Primary congenital glaucoma outcomes: lessons from 23 years of follow-up. Am J Ophthalmol 2015;159(4):788-796. DOI: 10.1016/j.ajo.2015.01.019. 
11. de Silva DJ, Khaw PT, Brookes JL. Long-term outcome of primary congenital glaucoma. J Am Assoc Pediatr Ophthalmol Strabismus 2011;15(2):148-152. DOI: 10.1016/j.jaapos.2010.11.025.

12. Fung DS, Roensch MA, Kooner KS, et al. Epidemiology and characteristics of childhood glaucoma: results from the Dallas glaucoma registry. Clin Ophthalmol 2013;7:1739-1746. DOI: 10.2147/ OPTH.S45480.

13. Aponte EP, Diehl N, Mohney BG. Medical and surgical outcomes in childhood glaucoma: a population-based study. J AAPOS Off Publ Am Assoc Pediatr Ophthalmol Strabismus 2011;15(3):263-267. DOI: 10.1016/j.jaapos.2011.02.015.

14. Khalil DH, Abdelhakim MASE. Primary trabeculotomy compared to combined trabeculectomy-trabeculotomy in congenital glaucoma: 3-year study. Acta Ophthalmol 2016;94(7):e550-e554. DOI: 10.1111/ aos.13018.

15. Cup-to-Disc Ratio - an overview | ScienceDirect Topics [Internet]. [cited 2019 May 30]. Available from: https://www.sciencedirect.com/ topics/medicine-and-dentistry/cup-to-disc-ratio.

16. Jalil A, Au L, Khan I, et al. Combined trabeculotomy-trabeculectomy augmented with 5-fluorouracil in paediatric glaucoma. Clin Exp Ophthalmol 2011;39(3):207-214. DOI: 10.1111/j.14429071.2010.02444.x.

17. Liu D, Huang L, Mukkamala L, et al. The economic burden of childhood glaucoma. J Glaucoma 2016;25(10):790-797. DOI: 10.1097/ IJG.0000000000000412 